\title{
Surveillance of donated blood during the 2016 arbovirus outbreak in Brazil
}

\author{
Rohit Sharma ${ }^{1}$ | Larissa Costa Santos ${ }^{1}$ | Robson A. da Silva ${ }^{2}$ | \\ Caroline V. Gonçalves ${ }^{2}$ | Stéfano de Melo Calado ${ }^{2}$ | Denisar P. Santos ${ }^{2}$ | \\ Josana P. de Andrade de Melo ${ }^{3}$ | Rita de Cássia Pontello Rampazzo 4 | \\ Luciana Requião $^{4}$ | Marco A. Krieger ${ }^{4,5}$ | Manoel Barral-Netto ${ }^{1,6}$ | \\ Camila I. de Oliveira ${ }^{1,6}$
}

${ }^{1}$ Instituto Gonçalo Moniz-IGM/FIOCRUZ, Salvador, BA, Brazil

2 IMS-Instituto Multidisciplinar em Saúde, UFBA, Vitória da Conquista, BA, Brazil

${ }^{3}$ Serviço de Hemoterapia do Sudoeste, Vitória da Conquista, BA, Brazil

${ }^{4}$ IBMP-Instituto de Biologia Molecular do

Paraná, Curitiba, PR, Brazil

5 Instituto Carlos Chagas-ICC/FIOCRUZ,

Curitiba, PR, Brazil

${ }^{6}$ Instituto Nacional de Ciência e Tecnologia de Investigação em Imunologia, São Paulo, SP, Brazil

\section{Correspondence}

Camila I. de Oliveira, Instituto Gonçalo MonizIGM-FIOCRUZ, Rua Waldemar Falcão, 121, Candeal Salvador, BA 40196-710, Brazil.

Email: camila@bahia.fiocruz.br

Funding information

CAPES Fellowship
Reports of arboviral transmission via blood transfusion may be a cause of concern among asymptomatic infected donors. This study evaluated the presence of arboviruses in donated blood products during the 2016 outbreak in Vitória da Conquista (Bahia-Brazil). Serum samples ( $n=676$ ) were screened for ZIKV, CHIKV, and the four DENV serotypes using a one-step reverse transcriptase-based Real-Time Polymerase Chain Reaction (RT-PCR). No samples tested positive for any of the targets, whereas positive controls performed as expected. The results suggest a low risk of arboviral transmission via transfusion of blood products in the endemic area studied during the 2016 outbreak.

\section{KEYWORDS}

NAT, transfusion, ZIKV

\section{1 | INTROCUCTION}

Arthropod-borne viral infections, highly prevalent in tropical and subtropical countries, inflict millions of people every year. Brazil, in particular, has witnessed periodic dengue virus (DENV; Flavivirus) epidemics since 1981 and, more recently, the appearance of both chikungunya (CHIKV; Alphavirus) and Zika viruses (ZIKV; Flavivirus) has raised public health concerns. ${ }^{1,2}$ Most ZIKV infections are asymptomatic or result in mild febrile disease with rash and conjunctivitis but its association with microcephaly in newborns resulted in a national public health emergency in Brazil. $^{3}$ DENV causes flu-like illness, and occasionally develops into severe dengue (www.who.int/factshet). CHIKV causes fever and severe joint pain; other symptoms include muscle pain, headache, nausea, fatigue, and, most strikingly, neonatal neurological disorders. ${ }^{4}$ Since all three viruses share a common vector, Aedes aegypti, it has been suggested that co-circulation in affected areas may impact the observed clinical outcomes. ${ }^{5}$

In addition to the clinical implications of arboviral infection, transfusion transmission is also a concern due to short periods of asymptomatic viremia. Viral transmission via blood transfusion has been reported for DENV, ${ }^{6}$ West Nile Virus, ${ }^{7}$ and $Z$ ZIKV. ${ }^{8}$ In a recent ZIKV outbreak in French Polynesia, 2.8\% of blood donors tested positive for ZIKV using nucleic acid testing (NAT). ${ }^{9}$ Although ZIKVreactive blood products were indeed transfused to blood recipients, no post-transfusion symptomatic ZIKV infections were reported. A larger study that also used NAT was performed in over 450000 blood 
donations in the United States, with five cases testing positive for ZIKV RNA. Again, no instances of post-transfusion infection were observed, despite the probable transfusion of low levels of ZIKV RNA, suggesting that blood units may present a low risk of infection. ${ }^{10}$ In Brazil, a report of ZIKV transmission by platelet transfusion ${ }^{11}$ confirmed the identity of a ZIKV isolate in the donor and two recipients by molecular sequencing accompanied by phylogenetic analysis of RNA, which strongly points to transfusion as the source of infection. Once again, none of the transfused patients reported any symptoms compatible with ZIKV.

Given previous reports of arboviral RNA detected in donated blood units, this study sought to investigate the presence of arboviruses (DENV 1-4, CHIKV, and ZIKV) in blood units donated to the municipal blood bank of Vitória da Conquista, located in BahiaBrazil, during the outbreak of 2016. A total of 676 serum samples were obtained during 2016 and tested using a one-step reverse transcriptase Real-time PCR assay.

\section{2 | MATERIALS AND METHODS}

\subsection{Study design and population}

The purpose of this study was to screen for asymptomatic infection in 676 units of blood donated to the Serviço de Hemoterapia do Sudoeste, located in Vitória da Conquista, Bahia-Brazil, over a period of 3 months (April to June 2016). Vitória da Conquista is situated in the southwestern part of Bahia, Brazil, with a population estimated in 348718 inhabitants (IBGE-2017) and is the third largest city in the state. This research received approval from the Institutional Review Board of the Gonçalo Muniz Institute (Salvador, Bahia, Brazil; protocol no. 1.778.639) and the Comissão Nacional de Ética em Pesquisa (CEP, Brazilian National Ethics Committee, Brazil). Informed written consent was obtained from each participant. All methods were performed in accordance with the guidelines and regulations determined by the CEP.

\section{2 | Sample collection}

Whole blood was collected in quadruple blood packs (Fresenius/KAB), using the top-and-bottom system. Aliquots of $5 \mathrm{~mL}$ of peripheral blood were obtained following blood collection and placed into Vacutainer ${ }^{\circledR}$ tubes (Becton Dickinson, Franklin Lakes, NJ) containing heparin as an anti-coagulant. Serum was separated by centrifugation and stored at $-70^{\circ} \mathrm{C}$ for subsequent RNA extraction (aliquots of $140 \mu \mathrm{L}$ ) using a QIAmp viral RNA mini kit (Qiagen, Hilden, Germany) in accordance with manufacturer instructions. RNA was eluted at a final volume of $60 \mu \mathrm{L}$ and frozen at $-70^{\circ} \mathrm{C}$ until the time of use.

\section{3 | Nucleic acid testing}

Arboviral RNA was detected by nucleic acid testing (NAT) using a ZDC diagnostic kit (Kit Biomol ZDC-IBMP, Instituto Biologia Molecular do Paraná, Brazil), which employs a one-step TaqMan-based RT-PCR assay for the qualitative detection of ZIKV, DENV (serotype specific), and

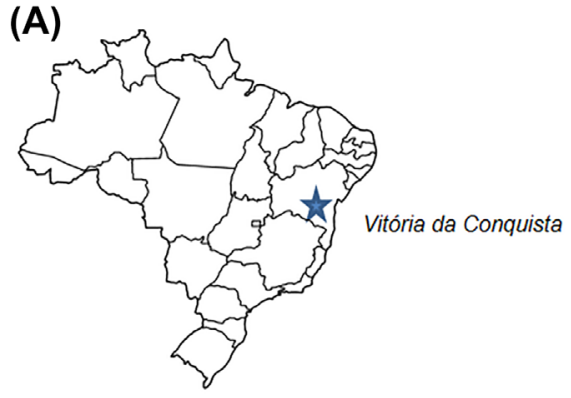

(B)

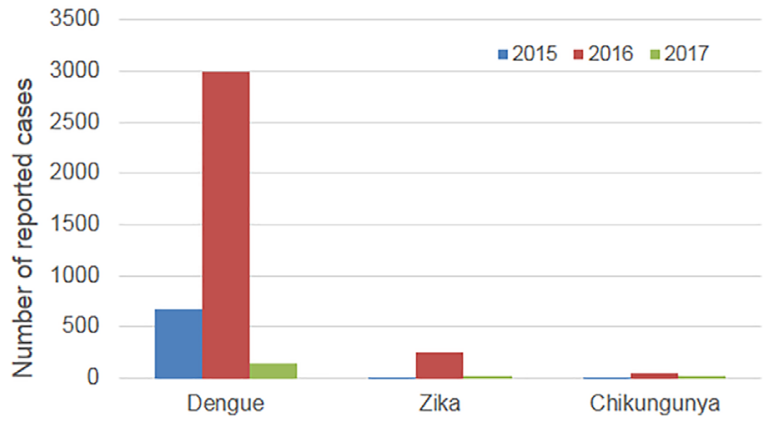

FIGURE 1 Study site and number of clinical cases. A, Map of Brazil locating the study site, Vitoria de Conquista, southwestern Bahia, Brazil. B, Number of cases of ZIKV, DENV, and CHIKV for the years 2015-2017 in Vitória da Conquista, Bahia, Brazil (Secretaria de Saúde do Estado da Bahia)

CHIKV RNA. This kit supplies internal controls for amplification of a human target to ensure extracted RNA quality. Amplification was performed using $9.5 \mu \mathrm{L}$ of extracted RNA as a template in a $20 \mu \mathrm{L}$ final volume PCR reaction to detect ZIKV, CHIKV and DENV (serotypes 1-4) using a 7500 Real-Time PCR Instrument (Applied Biosystems, Foster

\section{Experimental design}

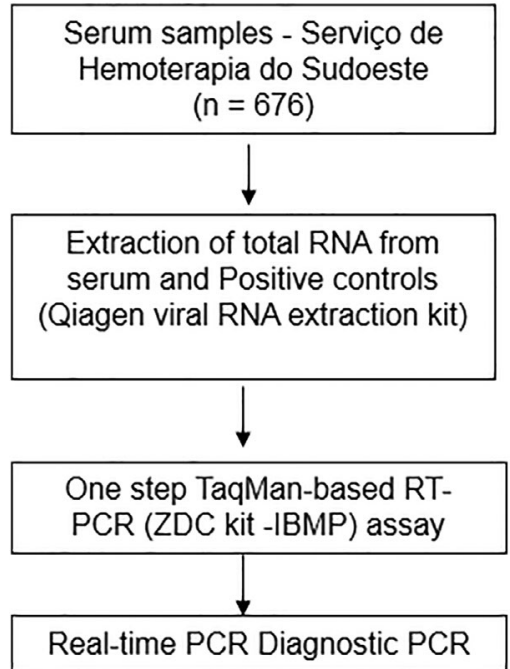

FIGURE 2 Experimental design showing sample collection and processing 
City, CA) following the manufacturer's protocol: a single cycle at $51^{\circ} \mathrm{C}$ for $30 \mathrm{~min}$, followed by another at $95^{\circ} \mathrm{C}$ for $15 \mathrm{~min}$, then 40 cycles of $95^{\circ} \mathrm{C}$ for $15 \mathrm{~s}$, each subsequently followed by $60^{\circ} \mathrm{C}$ for $1 \mathrm{~min}$. Positive controls supplied by the manufacturer were used, in addition to positive samples from patients diagnosed with CHIKV (kindly donated by $\mathrm{Dr}$
Ricardo Khouri) and viral stocks obtained from infected cells (DENV-142735/BR-PE/97; DENV-2-3808/BR-PE/95; DENV-3-95016/BR-PE/ 02; DENV-4-0081/BR-PE; ZIKV-PE243/2015) (kindly donated by Drs Rafael Dhalia and Ernesto Marques). Ct values <36 were deemed positive and values between 36 and 40 were considered indeterminate.

(A)

\section{ZIKV}

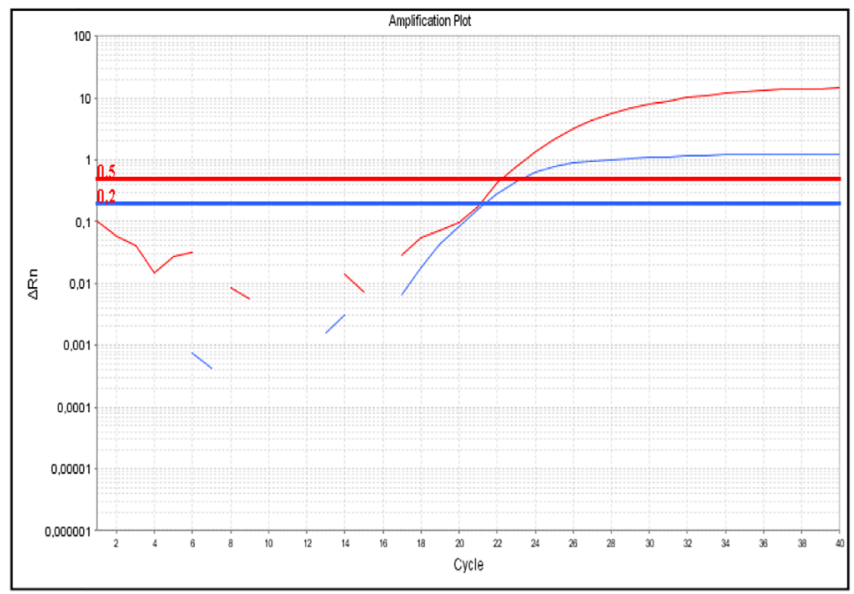

DENV $(1,4)$

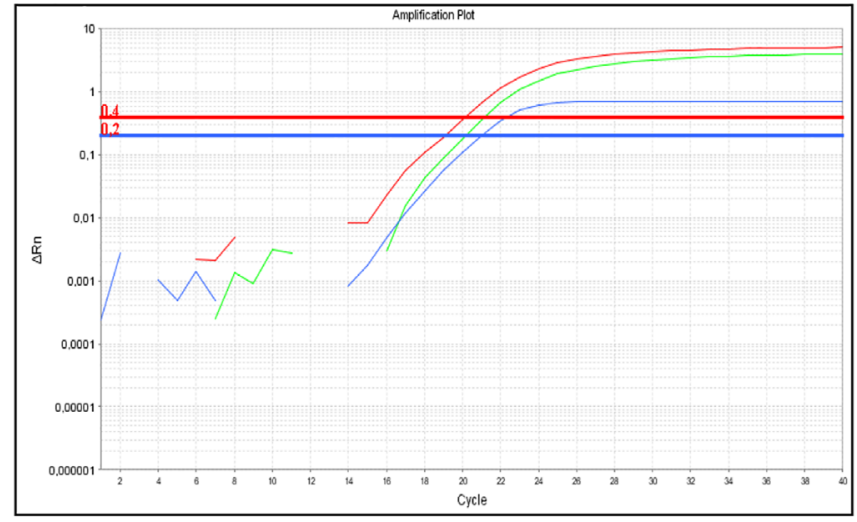

(B)

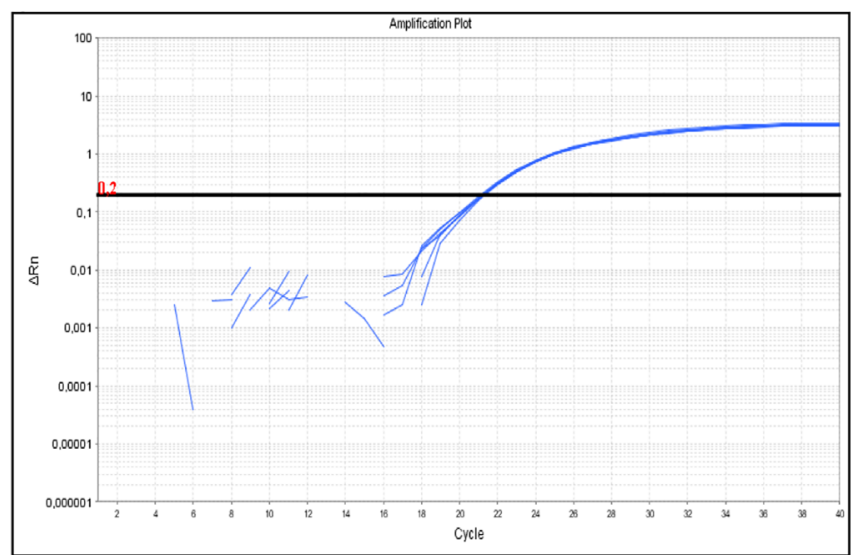

CHIKV

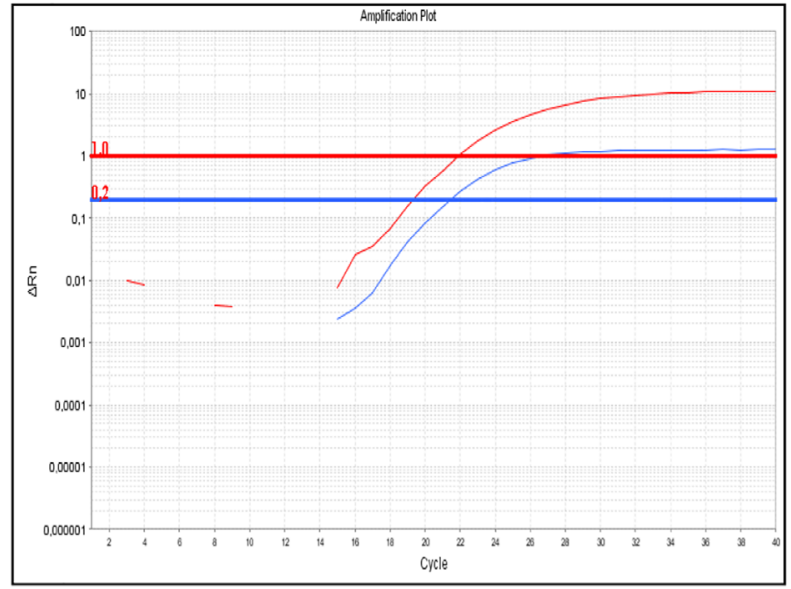

DENV $(2,3)$

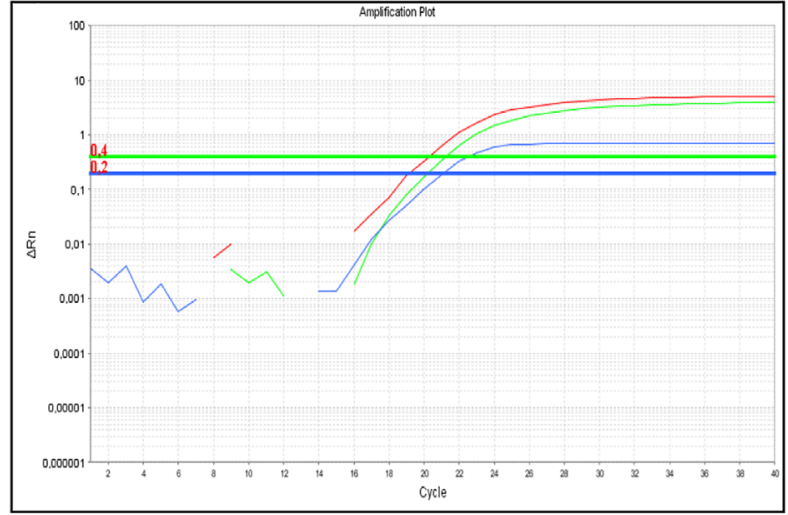

FIGURE 3 ZDC kit showing amplifications profiles for the targeted viruses. A, Amplification profiles depicting the Cts for positive controls (ZIKV, CHIKV, and DENV) supplied in the ZDC Kit, red line, and internal control, blue line. For DENV 1-4, positive controls are depicted in the red and green lines. B, Amplification profile of a representative negative sample depicting amplification of internal control only 


\section{3 | RESULTS}

In the year 2016, Vitória de Conquista, situated in southwestern Bahia, Brazil (Figure 1A) demonstrated an increase in the number of reported/ confirmed cases of DENV and ZIKV in comparison to 2015, as reported by Secretaria de Saúde da Bahia (SESAB) (Figure 1B). In 2017, the number of cases reported for the three arboviruses diminished considerably.

We thus screened 676 serum samples from healthy blood donors attending the Serviço de Hemoterapia do Sudoeste to determine the presence of arboviruses (DENV-1-4, CHIKV, and ZIKV) using nucleic acid testing (Figure 2A). RNA was extracted from experimental samples and from positive controls. Samples were thus submitted to TaqMan-based Real Time amplification of ZIKV, CHIKV, DENV-1-4 (Figure 2). The kit also supplies oligonucleotides for the detection of ZIKV, CHIKV, and DENV (1-4), including positive control and internal control. Representative amplification plots are depicted in Figure 3. None of the samples obtained from the Serviço de Hemoterapia do Sudoeste tested positive for any of the targets (ZIKV, CHIKV, or DENV [1-4]). We also tested truly positive samples consisting of RNA obtained from clinically diagnosed patients (ZIKV and CHIKV) or from viral stocks obtained from DENV infected cells which behaved as expected (data not shown). These results indicate that, despite an increase in the number of DENV and ZIKV in Vitória da Conquista, during the years of 2015 and 2016, arboviral presence was not detected in blood donors attending the Serviço de Hemoterapia do Sudoeste.

\section{4 | DISCUSSION}

Brazil has witnessed DENV, CHIKV, and ZIKV outbreaks and faced challenges with respect to public health implications. In addition to adopting control measures for these infections, it is necessary to understand the potential risk posed by non-vector transmission, that is, blood transfusion. This study investigated the presence of arboviral RNA in donated blood units at the municipal blood bank in Vitória da Conquista (Bahia-Brazil), where ZIKV, DENV, and CHIKV co-circulated during the 2016 outbreak.

Following the screening of 676 blood samples, we did not detect any positive samples for ZIKV, DENV, or CHIKV, despite a number of cases reported, during the screening period. Our negative results differ from those reported by Musso et al on the potential for ZIKV transmission during the French Polynesia outbreak. The authors screened 1505 blood donors and found 42 (3\%) samples positive for ZIKV by PCR, although all infected individuals were asymptomatic at the time of donation. ${ }^{8}$ In light of this transfusion-transmitted ZIKV alert, subsequently published studies investigated the possibility of inactivation of ZIKV in blood components ${ }^{12-14}$ and addressed guidelines for the prevention of ZIKV transmission via transfusion. ${ }^{15}$ Nonetheless, ZIKV-positive blood donations have been reported in the United States in blood donors who traveled to endemic areas or had been sexually exposed prior to donation. ${ }^{16}$ These findings were corroborated in a different study, in which transmission of ZIKV to individuals receiving blood products was not detected, suggesting that the blood units harboring ZIKV RNA were not infectious. ${ }^{10}$

In a recent study conducted in Ribeirão Preto, northeastern part of state of São Paulo, Brazil, 1393 blood samples were screened for ZIKV using an in-house molecular test followed by anti-ZIKV IgM or IgG in positive samples. ${ }^{17}$ ZIKV was detected in $2.7 \%$ samples, with $70.3 \%$ of these presenting a viral load of approximately 1000 copies $/ \mathrm{mL}$. Indeed, in the study by Slavov et al, 5319 suspected ZIKV cases were reported by the Municipal Edpidemiological Surveillance Agency compared to 258 suspected cases herein. We speculate that the high prevalence reported by these two studies, in comparison to our negative results, may be related to a high number of cases in the studied population, making it more likely to detect asymptomatic carriers.

An investigation of transfusion-transmitted DENV Brazil found that DENV 4 viremia was confirmed in $0.51 \%$ of 40000 blood donors, and that the transfusion of DENV-positive components did not yield significant clinical differences. ${ }^{18}$ As for $\mathrm{CHIKV}$, viral RNA was detected in $0.36 \%$ (Guadeloupe) and $0.42 \%$ (Martinique) of 16386 blood donors during the $2014-2015$ outbreak, ${ }^{19}$ showing the risk of collecting blood from asymptomatic viremic patients during outbreaks. Despite the higher number of DENV cases reported in our study area, compared to ZIKV and CHIKV, we did not detect positive samples in blood donors, suggesting that this risk is minimized.

In conclusion, our study suggests that the risk of arbovirus transmission via transfusion is not necessarily present in areas with reported arbovirus circulation. However, in areas of high prevalence, contaminated blood products were successfully detected, highlighting the need for active surveillance strategies, especially in the face of emerging outbreaks in Brazil.

\section{ACKNOWLEDGMENTS}

The authors acknowledge Ricardo Khouri (IGM-FIOCRUZ-BA), Rafael Dhalia, and Ernesto T.A. Marques (IAM-FIOCRUZ-PE) for kindly donating samples positive for ZIKV, CHIKV, and cells infected with DENV viral stocks, all used as positive controls in the present study. RS was supported by a CAPES fellowship. MK, MBN, and $\mathrm{ClO}$ are senior investigators from CNPq.

\section{ORCID}

Camila I. de Oliveira iD http://orcid.org/0000-0002-7868-5164

\section{REFERENCES}

1. Azevedo Rdo S, Oliveira CS, Vasconcelos PF. Chikungunya risk for Brazil. Rev Saude Publica. 2015;49:58.

2. Campos GS, Bandeira AC, Sardi SI. Zika virus outbreak, Bahia, Brazil. Emerg Infect Dis. 2015;21:1885-1886.

3. Barreto ML, Barral-Netto M, Stabeli R, et al. Zika virus and microcephaly in Brazil: a scientific agenda. Lancet. 2016;387: 919-921. 
4. Johansson MA, Mier YT-RL, Reefhuis J, Gilboa SM, Hills SL. Zika and the risk of microcephaly. N Engl J Med. 2016;375:1-4.

5. Donalisio MR, Freitas ARR, Zuben A. Arboviruses emerging in Brazil: challenges for clinic and implications for public health. Rev Saude Publica. 2017;51:30.

6. Stramer SL, Linnen JM, Carrick JM, et al. Dengue viremia in blood donors identified by RNA and detection of dengue transfusion transmission during the 2007 dengue outbreak in Puerto Rico. Transfusion. 2012;52:1657-1666.

7. Pealer LN, Marfin AA, Petersen LR, et al. Transmission of West Nile virus through blood transfusion in the United States in 2002. N Engl J Med. 2003;349:1236-1245.

8. Musso D, Nhan T, Robin E, et al. Potential for Zika virus transmission through blood transfusion demonstrated during an outbreak in French Polynesia, November 2013 to February 2014. Euro Surveill. 2014; 19(14): pii 20761.

9. Bierlaire D, Mauguin S, Broult J, Musso D. Zika virus and blood transfusion: the experience of French Polynesia. Transfusion. 2017;57:729-733.

10. Williamson PC, Linnen JM, Kessler DA, et al. First cases of Zika virusinfected US blood donors outside states with areas of active transmission. Transfusion. 2017;57:770-778.

11. Motta IJ, Spencer BR, Cordeiro da Silva SG, et al. Evidence for transmission of zika virus by platelet transfusion. N Engl J Med. 2016;375:1101-1103

12. Aubry M, Richard V, Green J, Broult J, Musso D. Inactivation of Zika virus in plasma with amotosalen and ultraviolet A illumination. Transfusion. 2016;56:33-40.

13. Laughhunn A, Santa Maria F, Broult J, et al. Amustaline (S-303) treatment inactivates high levels of Zika virus in red blood cell components. Transfusion. 2017;57:779-789.
14. Santa Maria F, Laughhunn A, Lanteri MC, Aubry M, Musso D, Stassinopoulos A. Inactivation of Zika virus in platelet components using amotosalen and ultraviolet A illumination. Transfusion. 2017:57:2016-2025.

15. Vasquez AM, Sapiano MR, Basavaraju SV, Kuehnert MJ, RiveraGarcia B. Survey of blood collection centers and implementation of guidance for prevention of transfusion-transmitted zika virus infection - Puerto Rico, 2016. MMWR Morb Mortal Wkly Rep. 2016;65:375-378

16. Galel SA, Williamson PC, Busch MP, et al. First zika-positive donations in the continental United States. Transfusion. 2017;57:762-769.

17. Slavov SN, Hespanhol MR, Rodrigues ES, et al. Zika virus RNA detection in asymptomatic blood donors during an outbreak in the northeast region of Sao Paulo State, Brazil, 2016. Transfusion. 2017;57(12):2897-2901.

18. Sabino EC, Loureiro P, Lopes ME, et al. Transfusion-transmitted dengue and associated clinical symptoms during the 2012 epidemic in Brazil. J Infect Dis. 2016;213:694-702.

19. Gallian P, Leparc-Goffart I, Richard P, et al. Epidemiology of chikungunya virus outbreaks in Guadeloupe and Martinique, 2014: an observational study in volunteer blood donors. PLoS Negl Trop Dis. 2017;11:e0005254.

How to cite this article: Sharma R, Costa Santos L, da Silva

RA, et al. Surveillance of donated blood during the 2016 arbovirus outbreak in Brazil. J Med Virol. 2018;90: 1406-1410. https://doi.org/10.1002/jmv.25193 